\title{
Intravenous injection of mesenchymal stem cell spheroids for efficient pulmonary delivery and prolonged in vivo survival
}

\author{
Yoshihiko Shimazawa ${ }^{1}$, Kosuke Kusamori ${ }^{1}$, Mari Tsujimura ${ }^{1}$, Kazunori Shimizu ${ }^{2}$, Satoshi \\ Konishi $^{3}$, and Makiya Nishikawa ${ }^{1}$ \\ ${ }^{1}$ Tokyo University of Science - Noda Campus \\ ${ }^{2}$ Nagoya University Graduate School of Engineering School of Engineering \\ ${ }^{3}$ Ritsumeikan University College of Science and Engineering Graduate School of Science \\ and Engineering
}

March 22, 2021

\begin{abstract}
Because of their excellent therapeutic potential, mesenchymal stem cells (MSCs) have been used as cell therapeutics for various diseases. However, the survival rate and duration of MSCs after transplantation are extremely low and short, respectively. To solve these problems, in this study, we prepared multicellular spheroids of MSCs and investigated their survival and function after intravenous injection in mice. The murine adipose-derived MSC line m17.ASC was cultured in agarose-based microwell plates to obtain size-controlled m17.ASC spheroids of an average diameter and cell number of approximately $170 \mu \mathrm{m}$ and 1100 cells/spheroid, respectively. The intravenously injected m17.ASC spheroids mainly accumulated in the lung and showed a higher survival rate than suspended m17.ASC cells during the experimental period of 7 days. m17.ASC spheroids efficiently reduced the lipopolysaccharide-induced increase in plasma concentrations of interleukin- 6 and tumor necrosis factor- $\alpha$. These results indicate that spheroid formation improved the pulmonary delivery and survival of MSCs, as well as their therapeutic potential against inflammatory pulmonary diseases.
\end{abstract}

\section{Introduction}

Mesenchymal stem cells (MSCs) possess excellent therapeutic properties, including the ability to exert antiinflammatory and immunosuppressive effects and to differentiate into tissue-specific cells. ${ }^{[1]}$ In addition, MSCs are self-proliferating and can be prepared on a large scale after isolation from a variety of tissues. ${ }^{[2,3]}$ Based on these superior features, MSCs have been used in the treatment of graft-versus-host disease, ${ }^{[4]}$ autoimmune diseases, ${ }^{[5]}$ and inflammatory diseases. ${ }^{[6]}$ Although the therapeutic usefulness of MSC transplantation in patients with these diseases has been demonstrated in some cases, many studies have revealed that the survival rate and duration of MSCs after transplantation are extremely low and short, respectively. ${ }^{[7]}$ Therefore, it is necessary to increase the survival of administered MSCs for more efficient MSC-based therapy.

Multicellular spheroids, which are three-dimensional cultured cell aggregates, are highly functionalized cells, because spheroid formation generally increases cellular function through extensive cell-cell interactions. ${ }^{[8]}$ It has been reported that spheroid formation upregulates the expression of transporters in cancer cells. ${ }^{[9]}$ Therefore, cancer cell spheroids have been used to screen for bioactive compounds, including anticancer agents. In addition, Bartosh et al. reported that multicellular spheroids derived from MSCs secrete large amounts of anti-inflammatory cytokines. ${ }^{[10]}$ Additionally, our previous studies using poly(N-isopropylacrylamide)-coated polydimethylsiloxane-based microwell plates showed that spheroid formation improved the survival of mouse insulinoma NIT-1 cells after transplantation in mice, ${ }^{[11]}$ enhanced albumin secretion from human hepatoblastoma HepG2 cells, ${ }^{[12]}$ and forcibly polarized mouse macrophage-like J774.1 cells to M1-type macrophages with 
tumoricidal activity. ${ }^{[13]}$ Furthermore, we demonstrated that compared to suspended mouse adenocarcinoma colon26 cells, multicellular spheroids of colon26 cells efficiently accumulated in the lung after intravenous injection in mice and established pulmonary tumor colonies. ${ }^{[14]}$ Therefore, we hypothesized that spheroid formation could increase the survival of MSCs after intravenous injection and improve their therapeutic effects in lung diseases.

Here, we investigated the survival and function of MSC spheroids after intravenous injection in mice. First, the murine adipose-derived MSC line m17.ASC transfected with NanoLuc luciferase (NLuc) was cultured in agarose-based microwell plates to obtain size-controlled m17.ASC spheroids. Thereafter, tissue distribution was evaluated by measuring NLuc activity after intravenous injection of m17.ASC spheroids or suspended m17.ASC cells. Finally, the anti-inflammatory effect of suspended m17.ASC cells or m17.ASC spheroids was evaluated in a lipopolysaccharide (LPS)-induced inflammatory mouse model.

\section{Materials and methods}

\subsection{Materials}

Dulbecco's modified Eagle's medium (DMEM) was purchased from Nissui Pharmaceutical (Tokyo, Japan). Trypsin-ethylenediaminetetraacetic acid (EDTA) solution (0.25\% trypsin and $1 \mathrm{mM}$ EDTA) and trypan blue were purchased from Nacalai Tesque (Kyoto, Japan). SYBR Green PCR Master Mix was obtained from Gibco Invitrogen (Carlsbad, CA, USA). Fetal bovine serum (FBS) was purchased from Thermo Fisher Scientific (Waltham, MA, USA). Agarose S was purchased from Nippon Gene (Toyama, Japan). Isoflurane was purchased from Fujifilm Wako Pure Chemical Industries (Osaka, Japan). Hygromycin B Gold was purchased from InvivoGen (San Diego, CA, USA). Hank's Balanced Salt solution (HBSS) was purchased from Sigma-Aldrich (St. Louis, MO, USA). All other chemicals were of the highest grade commercially available.

\subsection{Animals}

Male FVB/N mice (6-8 weeks old) were purchased from CLEA Japan Inc. (Tokyo, Japan). All animal experiments were conducted in accordance with the principles and procedures outlined in the National Institutes of Health Guide for the Care and Use of Laboratory Animals. The protocols for the animal experiments were approved by the Animal Experimentation Committee of the Tokyo University of Science.

\subsection{Cell culture}

The murine adipose-derived MSC line m17.ASC was purchased from DS Pharma Biomedical (Osaka, Japan) and grown in DMEM supplemented with $20 \%$ inactivated FBS and penicillin-streptomycin-glutamine mixed solution. Peritoneal macrophages were collected from male FVB/N mice. In detail, cold phosphate-buffered saline (PBS, $5 \mathrm{~mL}$ ) was injected into the abdominal cavity of euthanized FVB/N mice using a 26G needle and syringe, and the PBS containing peritoneal cells was collected after rubbing the abdomen. The collected PBS was centrifuged at $300 \times g$ for 3 min to sediment and obtain the peritoneal cells. The cells were grown in DMEM supplemented with $10 \%$ inactivated FBS and penicillin-streptomycin-glutamine mixed solution. After 24-h incubation, adherent cells were used as peritoneal macrophages. All cells were maintained in a humidified atmosphere containing $5 \% \mathrm{CO}_{2}$ at $37^{\circ} \mathrm{C}$.

\subsection{Preparation of agarose-based microwell plates}

Micropillar arrays fabricated using a 3D printing system (Objet Geometries, Rehovot, Israel) ${ }^{[15]}$ were used as molds for agarose-based microwell plates. Agarose was dissolved in distilled water at a final concentration of $3 \%(\mathrm{w} / \mathrm{v})$, heated in a microwave oven, and poured into the micropillar arrays. After gelation of the agarose solution by cooling for $30 \mathrm{~min}$ at approximately $20^{\circ} \mathrm{C}$, the micropillar arrays were removed from the agarose gel. The agarose-based microwell plate was cut with a cutter to adjust the size to fit a well in a six-well cell culture plate. Microwell plates in culture plates were sterilized by ultraviolet irradiation prior to cell seeding. The structures of the micropillar arrays and agarose-based microwell plates were imaged using a digital camera (IXY 200F, Canon, Tokyo, Japan) and BZ-9000 microscope (Keyence, Osaka, Japan). 


\subsection{Preparation of m17.ASC spheroids}

m17.ASC cells $\left(2 \times 10^{6}\right.$ cells $)$ suspended in a cell culture medium were added to an agarose-based microwell plate in a six-well plate, and the culture plates were shaken for $2 \mathrm{~h}$. The medium was then replaced with fresh medium, and the cells were incubated at $37{ }^{\circ} \mathrm{C}$ with rotary shaking at $60 \mathrm{rpm}$. At 24, 48, and $72 \mathrm{~h}$ of incubation, spheroids formed in microwells were collected using a micropipette.

\subsection{Evaluation of m17.ASC spheroids}

The diameter of the m17.ASC spheroids was measured by observation under a BZ-9000 microscope (Keyence) using the BZ-9000 analyzer software. The average diameter was calculated as the average diameter of 100 spheroids. The number and viability of cells in spheroids were evaluated by trypan blue staining after dispersing 20 spheroids in trypsin-EDTA solution.

\subsection{Transforming growth factor (TGF)- $\beta$ release from m17.ASC spheroids}

To evaluate cytokine release from m17.ASC spheroids, monolayered m17.ASC cells $\left(1.25 \times 10^{4}\right.$ cells $)$ or m17.ASC spheroids (11 spheroids: approximately $1.25 \times 10^{4}$ cells, 24 -h incubation) were seeded into a 12 well culture plate. After 24 and $48 \mathrm{~h}$ of incubation, the supernatant was collected and centrifuged at 2,500 rpm for 5 min to remove cell debris. The concentration of TGF- $\beta$ in the supernatant was measured using a TGF beta-1 Human/Mouse Enzyme-Linked Immunosorbent Assay kit (Thermo Fisher Scientific).

\subsection{Polarization of macrophages by co-culture with m17.ASC spheroids}

To evaluate the polarization of macrophages by co-culture with m17.ASC spheroids, monolayered m17.ASC cells $\left(1 \times 10^{5}\right.$ cells $)$ or m17.ASC spheroids (90 spheroids: approximately $1 \times 10^{5}$ cells) were added to the upper wells of a 12-well Transwell plate (Corning, NY, USA), and $1 \times 10^{5}$ peritoneal macrophages were seeded into the lower wells. After 24-h incubation, total RNA was extracted from peritoneal macrophages using Sepasol RNA I Super G (Nacalai Tesque). mRNA was reverse-transcribed to cDNA using qPCR RT Master Mix with gDNA Remover (Toyobo, Osaka, Japan). To quantify the gene of interest, cDNA samples were amplified using Thunderbird SYBR qPCR Mix (Toyobo) and specific primers with the CFX Connect Real-Time System (Bio-Rad Laboratories, Hercules, CA, USA). The primer sequences used were as follows: GAPDH (F) 5'-AGGTCGGTGTGAACGGATTTG-3', GAPDH (R) 5'-TGtaGaCCATGTAGTtGagGTCA-3'; Arg1 (F) 5'-TGGCTTGCGAGACGTAGAC-3', Arg1 (R) 5'GCTCAGGTGAATCGGCCTTTT-3'; Ym1 (F) 5'-TTATCCTGAGTGACCCTTCTAAG-3', and Ym1 (R) 5'-TCATTACCCTGATAGGCATAGG-3'. Fold changes in mRNA expression were calculated using the $\triangle \triangle \mathrm{CT}$ method. ${ }^{[16]}$ The results were normalized to GAPDH mRNA levels in the same samples.

\subsection{Establishment of the Nluc -expressing m17.ASC cells}

The Nluc sequence was inserted into the pEBMulti/Hyg vector to construct a plasmid encoding Nluc (pEBMulti-Nluc) as previously reported. ${ }^{[17]}$ In brief, the cDNA fragment ofNluc was amplified by PCR and digested using the restriction enzymes KpnI and EcoRV (New England Biolabs, Ipswich, MA, USA). Subsequently, the product was inserted into the multiple cloning site of the pEBMulti-Hyg vector using the same restriction enzymes and Ligation High (Toyobo). m17.ASC cells $\left(5 \times 10^{4}\right.$ cells) were seeded into a 48-well culture plate and cultured for $24 \mathrm{~h}$. The pEBMulti-Nluc plasmid was transfected into m17.ASC cells using Lipofectamine 3000 (InvivoGen). The transfected m17.ASC cells were cultured in DMEM containing $20 \%$ FBS for 3 days. The cells were then cultured in media containing $400 \mu \mathrm{g} / \mathrm{mL}$ hygromycin B to select Nluc -expressing m17.ASC (m17.ASC/Nluc) cells. Nlucexpression in cells was confirmed using an Nluc substrate (Nano-Glo Luciferase Assay Reagent, Promega, Madison, WI, USA). m17.ASC/Nluc cells were cultured in a medium containing $400 \mu \mathrm{g} / \mathrm{mL}$ hygromycin.

2.10. Tissue distribution of m17.ASC/Nluc spheroids after intravenous injection in mice

m17.ASC/Nluc spheroids (135 spheroids: approximately $1.5 \times 10^{5}$ cells) or suspended m17.ASC/Nluc cells $\left(1.5 \times 10^{5}\right.$ cells) were intravenously injected into $\mathrm{FVB} / \mathrm{N}$ mice. Blood and tissues (heart, lung, liver, kidney, and spleen) were collected $24 \mathrm{~h}$ after intravenous injection. Tissues were homogenized in a lysis buffer $(20$ 
$\mathrm{mM}$ Tris- $\mathrm{HCl}, 200 \mathrm{mM} \mathrm{NaCl}, 2.5 \mathrm{mM} \mathrm{MgCl}_{2}, 0.05 \%$ (w/v) NP-40, $\mathrm{pH} 7.4$ ) using a homogenizer (Microtec, Chiba, Japan). Blood and homogenized tissues were centrifuged at $10,000 \times g$ for 15 min at $4{ }^{\circ} \mathrm{C}$ to obtain plasma and the supernatant of homogenized tissues, respectively. Subsequently, the relative light units of the plasma and supernatants were measured using the Nano-Glo Luciferase Assay Reagent with an Envision multilabel plate reader (PerkinElmer, Waltham, MA, USA). In addition, m17.ASC/Nluc spheroids (100 spheroids, 24-h incubation: approximately $1.5 \times 10^{5}$ cells $)$ or suspended m17.ASC/Nluc cells $\left(1.5 \times 10^{5}\right.$ cells) were intravenously injected into FVB/ $\mathrm{N}$ mice. Lungs were collected at $6,24,48,120$, and $168 \mathrm{~h}$ after intravenous injection and homogenized in the lysis buffer. The homogenates were centrifuged at 10,000 $\times$ $g$ for 10 min at $4{ }^{\circ} \mathrm{C}$ to obtain the supernatant of the homogenized lungs. The relative light units of the supernatants were measured, as described above.

To image m17.ASC spheroids or suspended m17.ASC cells in the lung after intravenous injection, suspended m17.ASC cells $\left(1 \times 10^{5}\right.$ cells) or m17.ASC spheroids (90 spheroids, 24 -h incubation: approximately $1 \times 10^{5}$ cells) were labeled with carboxyfluorescein diacetate succinimidyl ester (CFSE, Sigma-Aldrich). Lungs were collected from euthanized FVB/N mice $24 \mathrm{~h}$ after intravenous injection and fixed using O.C.T. compound (Sakura Finetek Japan, Tokyo, Japan). Cryosections of the lung were prepared using a Leica CM3050 S cryostat (Leica Microsystems, Nussloch, Germany) and observed under a BZ-9000 microscope (Keyence).

\subsection{Effect of m17.ASC spheroids on the LPS-induced inflammatory mouse model}

LPS (Sigma-Aldrich) was intraperitoneally administered to FVB/N mice at a dose of $1 \mathrm{mg} / \mathrm{kg}$. The mice were then bred on a hot plate $\left(40{ }^{\circ} \mathrm{C}\right)$ for $1 \mathrm{~h}$. Subsequently, m17.ASC spheroids ( 850 spheroids, 24 -h incubation: approximately $1 \times 10^{6}$ cells $)$ or suspended m17.ASC cells $\left(1 \times 10^{6}\right.$ cells $)$ were intravenously injected in the mice. Blood was collected from each mouse at 6 and $10 \mathrm{~h}$ after the injection. Blood was centrifuged at 10,000 $\times g$ for 5 min at $4{ }^{\circ} \mathrm{C}$ to obtain plasma. The plasma levels of IL- 6 and TNF- $\alpha$ were measured using ELISA kits (Thermo Fisher Scientific).

\subsection{Statistical analysis}

The statistical significance of differences was evaluated by one-way analysis of variance (ANOVA) followed by Tukey's test for multiple comparisons or Student's $t$-test for two groups. Differences were considered statistically significant at $P<0.05$.

\section{Results}

\subsection{Preparation of m17.ASC spheroids}

Figure $1 \mathrm{~A}$ and $\mathrm{B}$ show the appearance of the micropillar arrays and an agarose-based microwell plate, respectively. Figure 1C and D show microscopic images of the typical top and cross-sectional views of the agarose-based microwell plate. m17.ASC spheroids were successfully prepared using the agarose-based microwell plate $24 \mathrm{~h}$ after seeding the cells (Figure 1E). Figure 2 shows the size histogram of m17.ASC spheroids collected at 24,48 , and $72 \mathrm{~h}$ after incubation. The average diameter of the m17.ASC spheroids was approximately $170 \mu \mathrm{m}$, irrespective of the incubation period (Table 1). The number of m17.ASC cells ranged from 1,100 to 1,300 cells/spheroid and remained almost unchanged during the incubation period. The viability of the cells in spheroids was $>90 \%$. Based on these results, m17.ASC spheroids prepared from 24-h incubation were used in subsequent experiments.

\subsection{Characteristics of m17.ASC spheroids}

To evaluate the function of m17.ASC spheroids, the secretion of TGF- $\beta$, an anti-inflammatory cytokine, from m17.ASC spheroids or monolayered m17.ASC cells was measured. TGF- $\beta$ secretion in both groups increased with time, and the amount of TGF- $\beta$ secreted from m17.ASC spheroids tended to be higher than that from monolayered m17.ASC cells (Figure 3A). In addition, the effect of m17.ASC spheroids on the polarization of macrophages was evaluated, because MSCs are reported to modulate macrophages to the immunosuppressive M2-type and contribute to suppressing inflammation. ${ }^{[18]}$ m17.ASC spheroids and monolayered m17.ASC cells tended to increase the expression of Arg1 and $Y m 1$, two M2-type macrophage 
markers, in peritoneal macrophages (Figure 3B). The expression of Arg1 in m17.ASC spheroids was higher than that in monolayered m17.ASC cells, although the difference was not statistically significant. These results indicate that spheroid formation enhanced the functions of m17.ASC cells, as previously reported in MSC spheroid formation. ${ }^{[19]}$

\subsection{Tissue distribution of m17.ASC/Nluc spheroids and suspended m17.ASC/Nluc cells in mice}

Figure 4A shows the tissue distribution of m17.ASC/Nluc spheroids and suspended m17.ASC/Nluc cells 24 $\mathrm{h}$ after intravenous injection in mice. m17.ASC/Nluc spheroids and suspended m17.ASC/Nluc cells showed high accumulation in the lung compared to other tissues. m17.ASC/Nluc spheroid accumulation in the lung was significantly higher than that in suspended m17.ASC/Nluc cells. Figure 4B shows the m17.ASC/Nluc cells remaining in the lung over time. At all the time points examined, the number of remaining cells was higher in the m17.ASC/Nluc spheroid group than that in the suspended m17.ASC/Nluc cell group. Furthermore, m17.ASC/Nluc spheroids were detected in the lung even at $168 \mathrm{~h}$ after injection, whereas suspended m17.ASC/Nluc cells almost completely disappeared after $48 \mathrm{~h}$. Figure $4 \mathrm{C}$ shows typical images of the cryosections of the lung at $24 \mathrm{~h}$ after intravenous injection of CFSE-labeled m17.ASC spheroids or suspended CFSE-labeled m17.ASC cells. The fluorescence signal of CFSE-labeled m17.ASC spheroids was observed in most sections examined, whereas the fluorescence signal of suspended CFSE-labeled m.17ASC cells was hardly detected in any sections.

\subsection{Effect of m17.ASC spheroids on LPS-induced inflammation in mice}

Figure 5 shows the plasma concentrations of IL-6 and TNF- $\alpha$ in the LPS-induced inflammation mouse model. m17.ASC spheroids and suspended m17.ASC cells significantly reduced the LPS-induced increase in IL-6 and TNF- $\alpha$ levels. Importantly, m17.ASC spheroids were more effective than suspended m17.ASC cells in reducing TNF- $\alpha$ levels at $6 \mathrm{~h}$. These results indicate that the therapeutic effect of m17.ASC spheroids was higher than that of suspended m17.ASC cells.

\section{Discussion}

Multicellular spheroids are useful cell formations in cell-based therapy because of the excellent cell-cell interactions, physical strength, and avoidance of anoikis, which is an apoptotic response to the absence of cell-matrix interactions. Although many reports have demonstrated improved cellular functions by spheroid formation in vitro, ${ }^{[20-22]}$ there have been few reports on the function or survival of multicellular spheroids after transplantation into animals. Our previous studies demonstrated that the survival of insulin-secreting cells was prolonged after transplantation into the subrenal capsule in mice ${ }^{[11]}$ and that tumor colony formation in the lungs after intravenous injection in mice was accelerated by spheroid formation of tumor cells. ${ }^{[14]}$ However, the function, survival, and tissue distribution of multicellular spheroids of MSCs after intravenous injection have been barely elucidated thus far. In particular, the quantitative comparison of suspended cells and spheroids after intravenous injection in terms of survival and tissue distribution has not been evaluated. Here, we showed that spheroid formation of MSCs improved their accumulation in the lungs and prolonged their survival after intravenous injection.

Several studies have reported that spheroid formation significantly increases cytokine release from MSCs. ${ }^{[10,23]}$ Here, TGF- $\beta$ release from m17.ASC spheroids was slightly higher than that from monolayered m17.ASC cells (Figure 3A). Additionally, the release of other cytokines from m17.ASC spheroids may have been improved, because the expression of Arg1, an M2-type marker, by peritoneal macrophages was greatly increased by co-culture with m17.ASC spheroids (Figure 3B). As the MSC-induced polarization of macrophages to the M2-type has been reported to be related to an anti-inflammatory function, ${ }^{[24]}$ the results of the present study strongly suggest the therapeutic potential of MSC spheroids for inflammatory diseases. In addition to cytokine expression, spheroid formation of MSCs can be an effective means of cell-based therapy.

The size of multicellular spheroids is an important parameter in determining the tissue distribution of cells after intravenous injection. This is because the diameter of microvessels in the lung, the first-pass organ 
after intravenous injection, is approximately $10 \mu \mathrm{m}$, and most cells transiently form the embolus in the lung after intravenous injection. ${ }^{[25]}$ Our previous study showed that intravenously injected multicellular spheroids of adenocarcinoma cells with a diameter of approximately $170 \mu \mathrm{m}$ effectively formed tumor colonies in the lung compared to suspended cells. ${ }^{[14]}$ Therefore, m17.ASC spheroids of approximately $170 \mu \mathrm{m}$ diameter were prepared in this study (Table 1), and intravenously injected m17.ASC spheroids efficiently accumulated and remained in the lung.

LPS-induced inflammatory mouse models are frequently used as systemic inflammatory disease models through endotoxin shock, which causes an increase in the release of inflammatory cytokines such as IL-6 in the plasma and multiple organ failures. ${ }^{[26]}$ Many reports have demonstrated that MSC administration effectively suppresses inflammation and shows a therapeutic effect in LPS-induced inflammatory mouse models. ${ }^{[27,28]}$ Compared to m17.ASC cells, m17.ASC spheroids more efficiently suppressed the plasma levels of inflammatory cytokines in LPS-induced inflammation mouse models (Figure 5). This result reflects the prolonged survival of the m17.ASC spheroids after intravenous injection (Figure 4).

In conclusion, we demonstrated that spheroid formation of m17.ASC cells improved the delivery efficiency of the cells to the lung and prolonged their survival after intravenous injection. Additionally, compared to suspended cells, the m17.ASC spheroids showed excellent therapeutic effect in the inflammatory mouse models.

\section{Acknowledgments}

The authors are grateful to Editage (www.editage.com) for English language editing. This work was supported in part by a GSK Japan Research Grant 2017.

\section{Conflict of interest}

The authors declare no competing interests.

\section{References}

1. J. Voswinkel, S. Francois, J. M. Simon, M. Benderitter, N.-C Gorin, M. Mohty, L. Fouillard, A. Chapel, Clin. Rev. Allergy Immunol.2013 , $45,180-192$.

2. N. Fekete, M. T. Rojewski, D. Fürst, L. Kreja, A. Ignatius, J. Dausend, H. Schrezenmeier, PLoS One . 2012,7 , e43255.

3. R. Hass, C. Kasper, S. Bohm, R. Jacobs, Cell Commun. Signal.2011, 9 , 12.

4. K. Muroi, K. Miyamura., M. Okada, T. Yamashita, M. Murata, T. Ishikawa, N. Uike, M. Hidaka, R. Kobayashi, M. Imamura, J. Tanaka, K. Ohashi, S. Taniguchi, T. Ikeda, T. Eto, M. Mori, M. Yamaoka, K. Ozawa,Int. J. Hematol. 2016 , 103, 243-250.

5. A. Tyndall, A. Houssiau, Ann. Rheum. Dis. 2010, 69, 1413-1414.

6. A. C. Inamdar, A. A. Inamdar, Exp. Lung Res. 2013, 39, 315-327.

7. L. Li, X. Chen, W. E. Wang, C. Zeng, Stem Cells Int.2016 , 2016, 9682757.

8. K. Kusamori, M. Nishikawa, N. Mizuno, T. Nishikawa, A. Masuzawa, Y. Tanaka, Y. Mizukami, K. Shimizu, S. Konishi, Y. Takahashi, Y. Takakura, Pharm. Res. 2016 , 33, 247-256.

9. H. Kim, Y. Phung, M. Ho, PLoS One 2012 , 7, e39556.

10. T. J. Bartosh, J. H. Ylostalo, A. Mohammadipoor, N. Bazhanov, K. Coble, K. Claypool, R. H. Lee, H. Choi, D. J. Prockop, Proc. Natl. Acad. Sci. USA 2010, 107, 13724-13729.

11. K. Kusamori, M. Nishikawa, N. Mizuno, T. Nishikawa, A. Masuzawa, K. Shimizu, S. Konishi, Y. Takahashi, Y. Takakura, J. Control. Release 2014, 173, 119-124.

12. T. Nishikawa, Y. Tanaka, M. Nishikawa, Y. Ogino, K. Kusamori, N. Mizuno, Y. Mizukami, K. Shimizu, S. Konishi, Y. Takahashi, Y. Takakura, Biol. Pharm. Bull., 2017 , 40, 334-338.

13. Y. Tanaka, M. Nishikawa, Y. Mizukami, K. Kusamori, Y. Ogino, S. Nishimura, K. Shimizu, S. Konishi, Y. Takahashi, Y. Takakura, J. Control. Release 2018, 28, 177-183.

14. T. Nishikawa., Y. Tanaka, K. Kusamori, N. Mizuno, Y. Mizukami, Y. Ogino, K. Shimizu, S. Konishi, Y. Takahashi, Y. Takakura, M. Nishikawa, Biotechnol. J. 2017, 12. 
15. K. Shimizu, K. Kusamori, M. Nishikawa, N. Mizuno, T. Nishikawa, A. Masuzawa, S. Katano, Y. Takahashi, Y. Takakura, S. Konishi, J. Biosci. Bioeng. 2013, 115, 695-699.

16. K. J. Livak, T. D. Schmittgen, Methods 2001, 25, 402-408.

17. M. Tsujimura, K. Kusamori, C. Oda, A. Miyazaki, H. Katsumi, T. Sakane, M. Nishikawa, A. Yamamoto, J. Control. Release 2018, 10, 78-84.

18. F. Braza, S. Dirou, V. Forest, V. Sauzeau., D. Hassoun, J. Chesné, M.-A. Cheminant-Muller, C. Sagan, A. Magnan, P. Lemarchand, Stem Cells 2016 , 34, 1836-1845.

19. C. Hoefner, C. Muhr, H. Horder, M. Wiesner, K. Wittmann, D. Lukaszyk, K. Radeloff, M. Winnefeld, M. Becker, T. Blunk, P. Bauer-Kreisel, Tissue Eng. Part A 2020 , 26, 915-926.

20. T. C. Tummy, M. Hughes-Fulfold, Tissue Eng. Part A2009 , 15, 559-567.

21. A. Ernst, S. Hofmann, R. Ahmadi, N. Becker, A. Korshunov, F. Engel, C. Hartmann, J. Felsberg, M. Sabel, H. Peterziel, M. Durchdewald, J. Hess, S. Barbus, B. Campos, A. Starzinski-Powitz, A. Unterberg, G. Reifenberger, P. Lichter, C. Herold-Mende, B. Radlwimmer. Clin. Cancer Res. 2009 , $15,6541-6450$.

22. S. C. Ramaiahgari, M. W. Braver, B. Herpers, V. Terpstra, J. N. M. Commandeur, B. van de Water, L. S. Price, Arch. Toxicol.2014, 88, 1083-1095.

23. L. H. Lee, Y. S. Han, S. H. Lee, Biomol. Ther. 2016 , 24, 260-267.

24. F. Zhang, H. Wang, X. Wang, G. Jiang, H. Liu, G. Zhang, H. Wang, R. Fang, X. Bu, S. Cai, J. Du, Oncotarget $2016,7,52294-52306$.

25. S. Schrepfer, T. Deuse, H. Reichenspurner, M. P. Fischbein, R. C. Robbins, M. P. Pelletier, Transplant. Proc. 2007, 39, 573-576.

26. D. G. Remick, D. E. Newcomb, G. L. Bolgos, D. R. Call, Shock2000 , 2, 110-116.

27. J. Xu, C. R. Woods, A. L. Mora, R. Joodi, K. L. Brigham, S. Iyer, M. Rojas, Am. J. Physiol. Lung Cell. Mol. Physiol. 2007 , 293, 131-141.

28. J. Li, D. Li, X. Liu, S. Tang, F. Wei, J. Inflamm. (Lond)2012, 9, 33.

Figure 1.

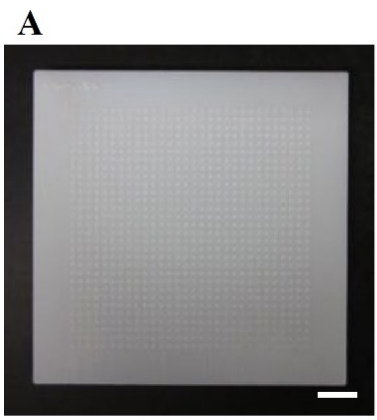

B

C
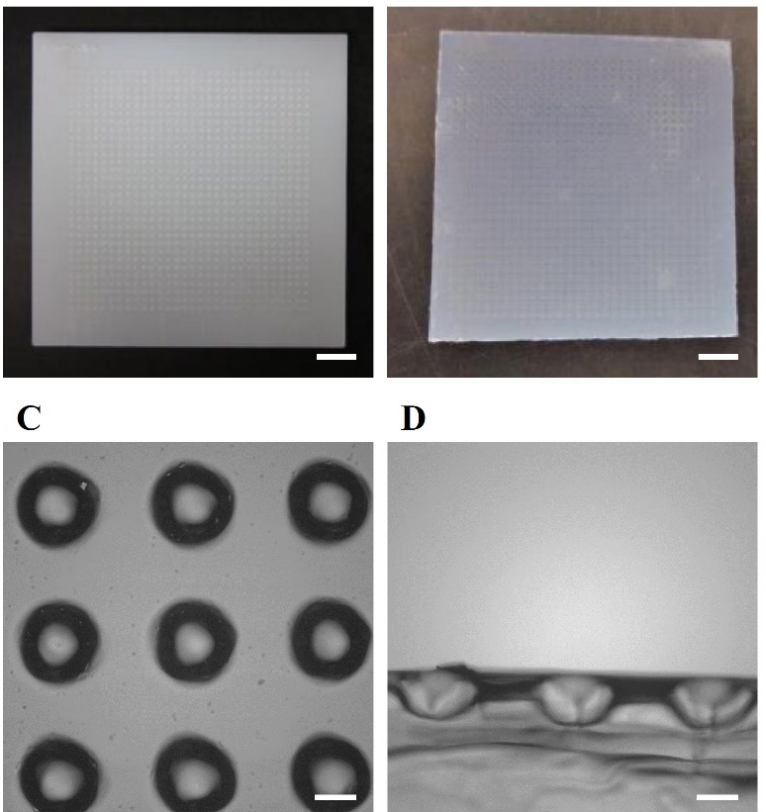

D

$\mathbf{E}$
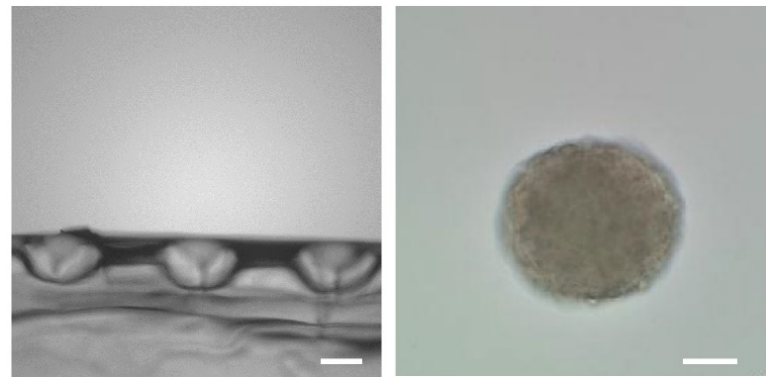

Figure 2. 

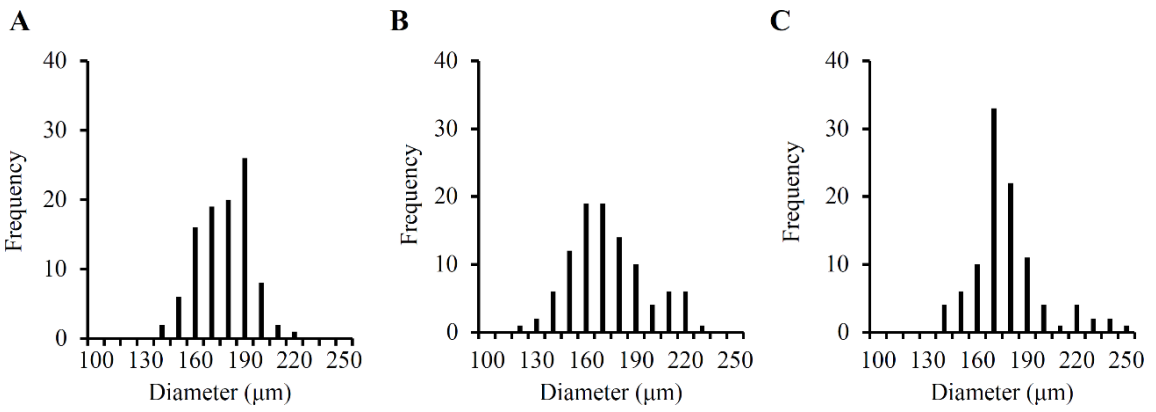

Figure 3.

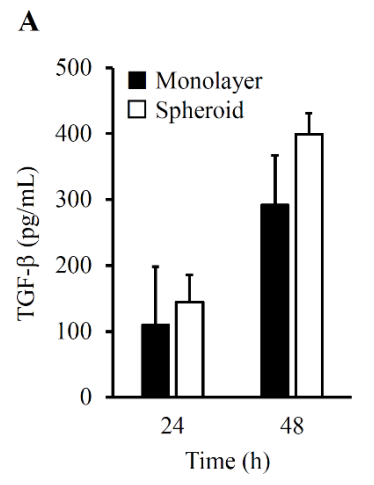

B
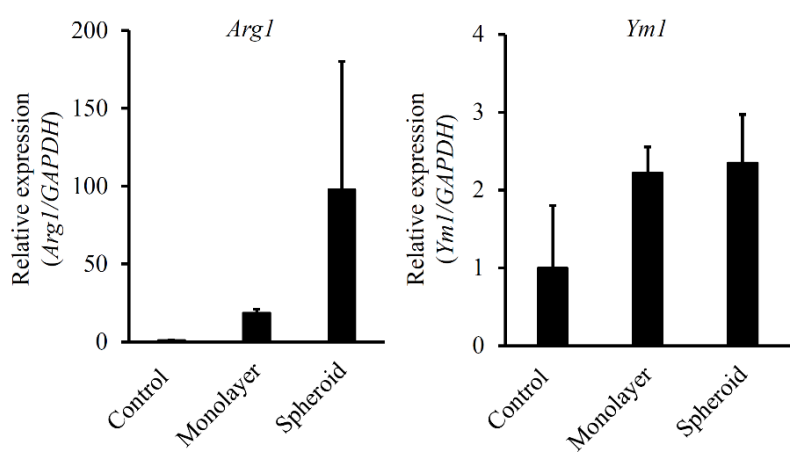

Figure 4 .
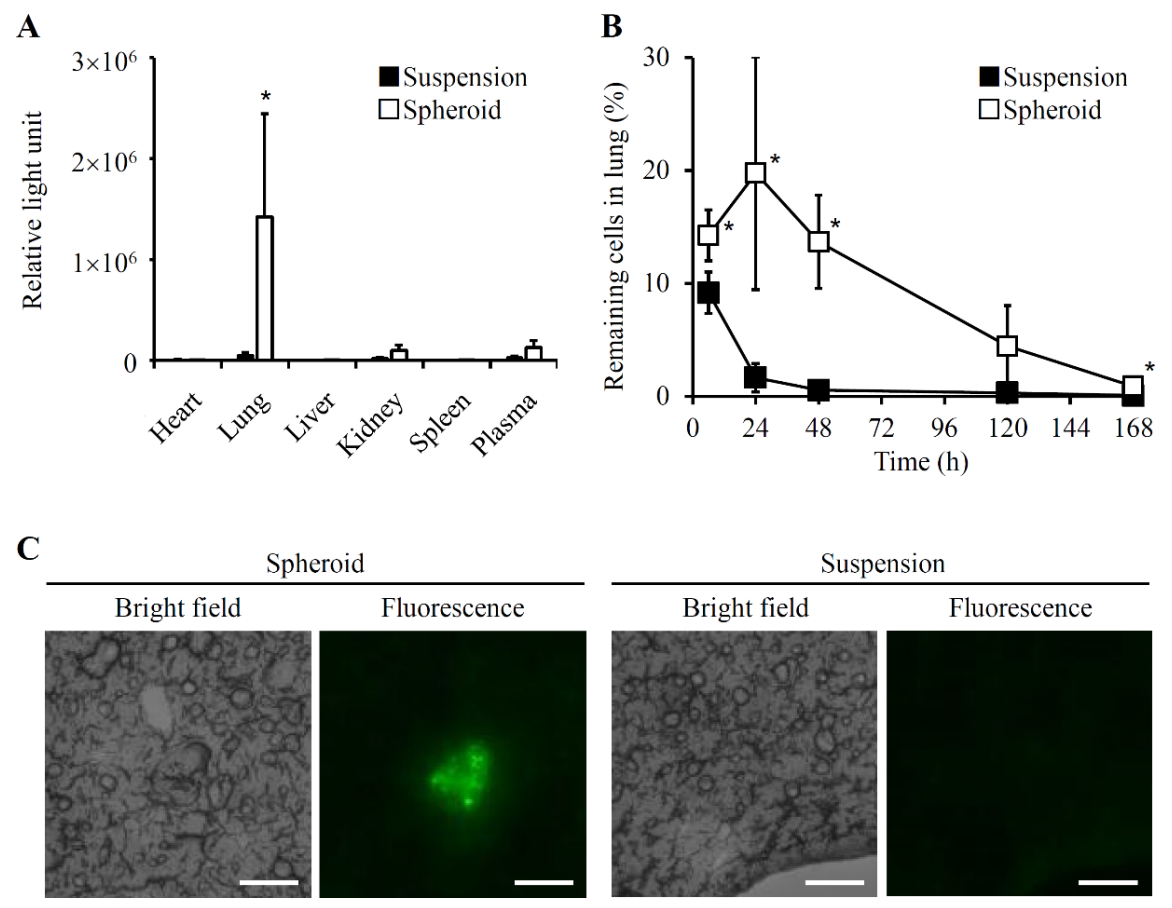

Figure 5. 
$\mathbf{A}$
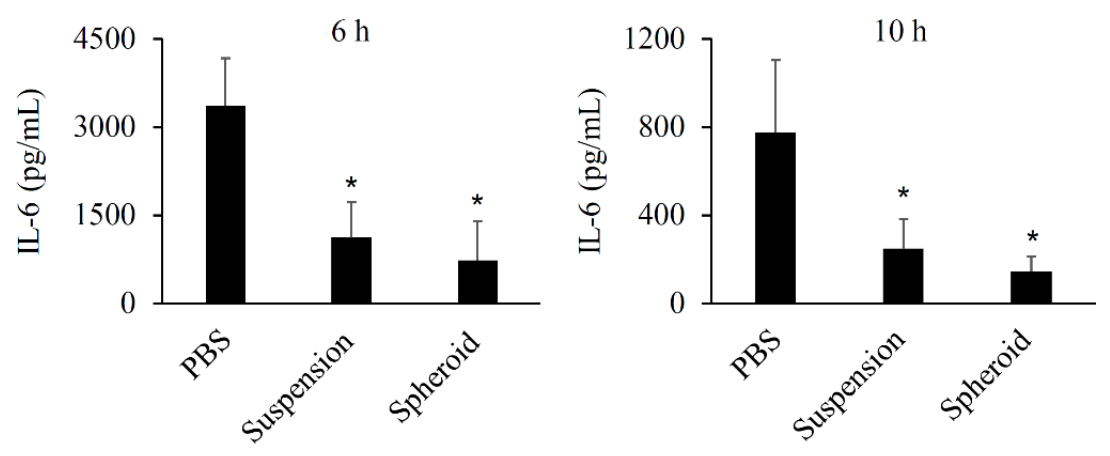

B
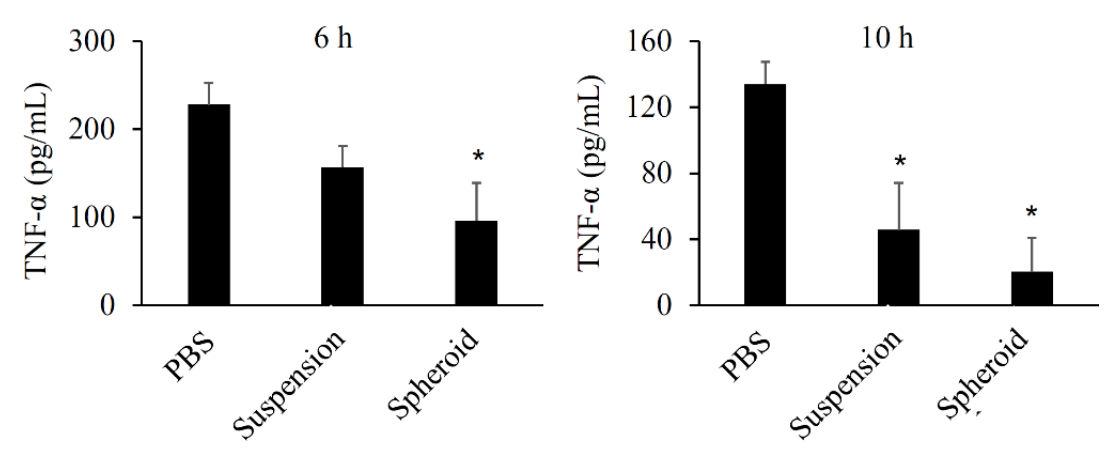

Table 1. Characteristics of m17.ASC spheroids

\begin{tabular}{llll}
\hline Time $(\mathrm{h})$ & Diameter $(\mu \mathrm{m})$ & Cell number/spheroid & Viability $(\%)$ \\
24 & $173 \pm 16$ & $1,110 \pm 90$ & $94.5 \pm 0.7$ \\
48 & $168 \pm 23$ & $1,300 \pm 90$ & $93.3 \pm 2.7$ \\
72 & $173 \pm 21$ & $1,170 \pm 60$ & $94.6 \pm 0.8$ \\
\hline
\end{tabular}

\section{Figure legends}

Figure 1. Preparation of m17.ASC spheroids. Macroscopic images of (A) micropillar arrays and (B) agarosebased microwell plate. Scale bars indicate $500 \mu \mathrm{m}$. (C) Top and (D) cross-sectional images of agarose-based microwell plate by microscopic observation. Scale bars indicate $300 \mu \mathrm{m}$. (E) A typical image of m17.ASC spheroid after 24-h incubation. Scale bar indicates $50 \mu \mathrm{m}$.

Figure 2. Size histogram of m17.ASC spheroids. m17. ASC spheroids were incubated for (A) 24 h, (B) 48 $\mathrm{h}$, and (C) $72 \mathrm{~h}$ after seeding cells in an agarose-based microwell plate. The diameters of 100 spheroids were measured using a BZ-9000 microscope.

Figure 3. Characteristics of m17.ASC spheroids. (A) TGF- $\beta$ secretion from m17.ASC spheroids. Monolayered m17.ASC cells $\left(1.25 \times 10^{4}\right.$ cells $)$ or m17.ASC spheroids (11 spheroids: $1.25 \times 10^{4}$ cells) were seeded into a 12-well culture plate. After 24- and 48-h incubation, the amount of TGF- $\beta$ in the supernatant was measured using an ELISA kit. (B) Arg1 and Ym1 expression in peritoneal macrophages after co-culture with m17.ASC spheroids. Monolayered m17.ASC cells $\left(1 \times 10^{5}\right.$ cells $)$ or m17.ASC spheroids $(90$ spheroids: $1 \times$ $10^{5}$ cells) were seeded into the upper wells of a 12 -well Transwell plate, and $1 \times 10^{5}$ peritoneal macrophages were seeded into the lower wells. After 24-h incubation, mRNA (Arg1 and Ym1) expression in peritoneal macrophages was evaluated using real time RT-PCR. Results are normalized to mRNA levels of GAPDH . Data are expressed as the mean $\pm \mathrm{SD}$ of three to four experiments. 
Figure 4. Biodistribution of m17.ASC/Nluc spheroids after intravenous injection. (A) Relative light units of m17.ASC/Nluc spheroids in the plasma and tissues. m17.ASC/Nluc spheroids (135 spheroids: $1.5 \times 10^{5}$ cells $/ 100 \mu \mathrm{L} /$ mouse $)$ or suspended m17.ASC/Nluc cells $\left(1.5 \times 10^{5}\right.$ cells $/ 100 \mu \mathrm{L} /$ mouse $)$ were intravenously injected in mice. Blood and tissues were collected at $24 \mathrm{~h}$ after intravenous injection, and relative light units of the plasma and supernatants of homogenized tissues were measured. (B) The numbers of m17.ASC/Nluc spheroids remaining in the lung. m17.ASC/Nluc spheroids (100 spheroids: $1.5 \times 10^{5}$ cells/mouse) or suspended m17.ASC/Nluc cells $\left(1.5 \times 10^{5}\right.$ cells/mouse $)$ were intravenously injected in mice. Lungs were collected at $6,24,48,120$, and $168 \mathrm{~h}$ after intravenous injection. The numbers of cells remaining in lungs were calculated by measuring the relative light units. (C) Typical images of CFSE-labeled m17.ASC spheroids in the lung. Suspended m17.ASC cells $\left(1 \times 10^{5}\right.$ cells $)$ or m17.ASC spheroids (90 spheroids: $1 \times 10^{5}$ cells) were labeled with CFSE. Lungs were collected from euthanized mice $24 \mathrm{~h}$ after intravenous injection of cells. Cryosections of the lung were observed using a BZ-9000 microscope. Scale bar indicates $100 \mu \mathrm{m}$. Data are expressed as the mean $\pm \mathrm{SD}$ of three to four experiments. ${ }^{*} P<0.05$; statistically significant differences compared with suspension group.

Figure 5. Effect of m17.ASC spheroids on LPS-induced inflammatory mouse model. LPS was intraperitoneally administered to mice at $1 \mathrm{mg} / \mathrm{kg}$. The mice were then bred on a hot plate $\left(40{ }^{\circ} \mathrm{C}\right)$ for $1 \mathrm{~h}$. $\mathrm{m} 17$.ASC spheroids (850 spheroids: $1 \times 10^{6}$ cells/100 $\mu \mathrm{L} /$ mouse $)$ and suspended m17.ASC cells $\left(1 \times 10^{6}\right.$ cells/100 $\mu \mathrm{L} /$ mouse) were intravenously injected. Blood was collected at 6 and $10 \mathrm{~h}$ after injection of cells. The (A) IL-6 and (B) TNF- $\alpha$ levels in plasma was measured using ELISA kits. Data are expressed as the mean \pm SD of three to four experiments. ${ }^{*} P<0.05$; statistically significant differences compared with the PBS group. 